\title{
Disfunción del esfínter de Oddi: un reporte de caso
}

\section{Sphincter of Oddi dysfunction: Case report}

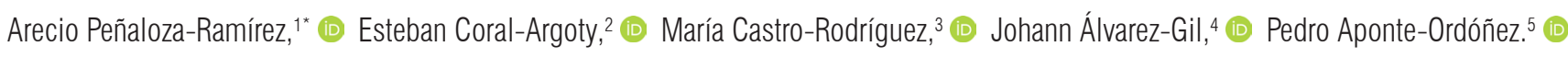

\begin{tabular}{|c|}
\hline $\begin{array}{l}\text { G ACCESO ABIERTO } \\
\text { Citación: } \\
\text { Peñaloza-Ramírez A, Coral-Argoty E, Castro- } \\
\text { Rodríguez M, Álvarez-Gil J, Aponte-Ordóñez } \\
\text { P. Disfunción del esfínter de Oddi: Un } \\
\text { reporte de caso. Rev Colomb Gastroenterol. } \\
\text { 2021;36(Supl.1):52-58. https://doi. } \\
\text { org/10.22516/25007440.550 }\end{array}$ \\
\hline $\begin{array}{l}1 \text { Investigador asociado, Colciencias. } \\
\text { Jefe, programa de especialización en } \\
\text { gastroenterología y endoscopia digestiva, } \\
\text { Fundación Universitaria Ciencias de la Salud, } \\
\text { Sociedad de Cirugía de Bogotá. Jefe, servicio } \\
\text { de gastroenterología y endoscopia digestiva, } \\
\text { Hospital de San José. Bogotá, Colombia. } \\
2 \text { Especialista en cirugía general. Residente de } \\
\text { gastroenterología y endoscopia digestiva, } \\
\text { Fundación Universitaria de Ciencias de la } \\
\text { Salud, Sociedad de Cirugía de Bogotá. Bogotá, } \\
\text { Colombia. } \\
3 \text { Especialista en medicina interna, residente } \\
\text { de gastroenterología y endoscopia digestiva, } \\
\text { Fundación Universitaria de Ciencias de la } \\
\text { Salud, Sociedad de Cirugía de Bogotá. Bogotá, } \\
\text { Colombia. } \\
{ }^{3} \text { Especialista en medicina interna. Residente } \\
\text { de gastroenterología y endoscopia digestiva, } \\
\text { Fundación Universitaria de Ciencias de la } \\
\text { Salud, Sociedad de Cirugía de Bogotá. Bogotá, } \\
\text { Colombia. } \\
5 \text { Especialista en cirugía general, } \\
\text { gastroenterología y endoscopia digestiva. } \\
\text { Instructor asistente, programa de } \\
\text { especialización en gastroenterología y } \\
\text { endoscopia digestiva, Fundación Universitaria } \\
\text { Ciencias de la Salud, Sociedad de Cirugía de } \\
\text { Bogotá. Bogotá, Colombia. }\end{array}$ \\
\hline $\begin{array}{l}\text { *Correspondencia: Arecio Peñaloza-Ramírez. } \\
\text { apenaloza@fucsalud.edu.co }\end{array}$ \\
\hline $\begin{array}{l}\text { Fecha recibido: } \quad 24 / 04 / 20 \\
\text { Fecha aceptado: } 26 / 10 / 20\end{array}$ \\
\hline
\end{tabular}

\section{Resumen}

La disfunción del esfínter de Oddi es un síndrome clínico causado por una enfermedad funcional (discinesia) o estructural (estenosis). La prevalencia estimada de disfunción del esfínter de Oddi en la población en general es del $1 \%$; aumentando a $20 \%$ para pacientes con dolor persistente posterior a colecistectomía y a $70 \%$ en pacientes con pancreatitis aguda recurrente idiopática. Se caracteriza clínicamente por la presencia de dolor abdominal, similar al cólico biliar o dolor tipo pancreático en ausencia de patología biliar orgánica; así como en pacientes con pancreatitis recurrente idiopática asociada con elevación de enzimas pancreáticas 0 hepáticas, y dilatación del conducto biliar o pancreático. El tratamiento para la disfunción del esfínter de Oddi tipo I se basa en la realización de esfinterotomía endoscópica, pero existe controversia en el manejo de la disfunción del esfínter de Oddi tipo II y III. En este artículo se presenta el caso clínico de una paciente de 67 años con antecedente de colecistectomía por laparotomía. Después del procedimiento quirúrgico refirió un dolor abdominal de predominio en el hipocondrio derecho tipo cólico asociado con emesis de características biliares. En el reporte de colangiorresonancia se encontró una ligera dilatación de la vía biliar intrahepática y gammagrafía con ácido iminodiacético hepatobiliar (HIDA) diagnóstica de disfunción del esfínter de Oddi. Se realizó una esfinterotomía endoscópica. En el seguimiento, dos años después, la paciente se encontraba asintomática con la disfunción del esfínter de Oddi resuelta.

\section{Palabras clave}

Discinesia del esfínter de Oddi, esfinterotomía endoscópica, gammagrafía, colangiopancreatografía retrógrada endoscópica.

\section{Abstract}

Sphincter of Oddi dysfunction is a clinical syndrome caused by functional (dyskinesia) or structural (stenosis) disease. The estimated prevalence of this condition in the general population is $1 \%$, reaching $20 \%$ in patients with persistent pain after cholecystectomy and $70 \%$ in patients with idiopathic recurrent acute pancreatitis. It is clinically characterized by the presence of abdominal pain, similar to biliary colic or pancreatic pain in the absence of organic biliary disease. It is also observed in patients with idiopathic recurrent pancreatitis, associated with elevated pancreatic or hepatic enzymes, and bile duct and/or pancreatic duct dilatation. Treatment for sphincter of Oddi dysfunction type I is based on endoscopic sphincterotomy, but there is controversy regarding the management of sphincter of Oddi dysfunction types II and III. This article presents the clinical case of a

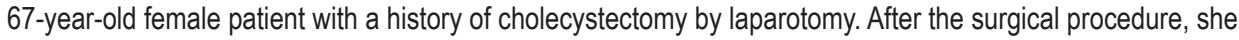
reported abdominal pain predominantly in the right hypochondrium, colicky, associated with emesis of biliary characteristics. Cholangioresonance report revealed mild intrahepatic bile duct dilatation, and scintigraphy with HIDA scan showed sphincter of Oddi dysfunction. Endoscopic sphincterotomy was performed. The patient was asymptomatic and the sphincter of Oddi dysfunction had resolved at two-year follow-up.

\section{Keywords}

Sphincter of Oddi dyskinesia; Endoscopic sphincterotomy; Scintigraphy; Endoscopic retrograde cholangiopancreatography. 


\section{INTRODUCCIÓN}

El esfínter de Oddi, descrito por primera vez por Ruggero Oddi en 1887 (1), es un sistema esfintérico compuesto por tejido conectivo, fibras circulares y longitudinales de músculo liso que en su mayoría se encuentran dentro de la pared duodenal. Se han descrito 3 zonas que componen el sistema esfintérico: esfínter del colédoco, esfínter pancreático y un esfínter común donde las fibras musculares de los dos esfínteres se entrelazan en forma de 8 (2).

El esfínter de Oddi se encarga de regular el flujo de bilis y la secreción exocrina del páncreas; previene el reflujo de contenido entérico desde el duodeno hacia el sistema pancreático y biliar; y entre comidas desvía la bilis hacia la vesícula biliar, lo que facilita su concentración fisiológica. En ayuno, el esfínter de Oddi mantiene presiones que oscilan entre $3 \mathrm{~mm} \mathrm{Hg}$ y $35 \mathrm{~mm} \mathrm{Hg}$ (se considera anormal cuando es $>40 \mathrm{~mm} \mathrm{Hg}$ ), con contracciones que promueven el flujo hacia el duodeno, con una frecuencia promedio de 4 por minuto y una duración promedio de 6 segundos. Aunque existe una relación con el complejo motor migratorio del duodeno, la mayor parte del flujo biliar parece ser pasivo entre las contracciones del esfínter de Oddi y aumentarse por la contracción de la vesícula biliar, la distensión antral, el efecto de la colecistoquinina y la disminución de la amplitud de las contracciones del esfínter de Oddi en el estado posprandial (3).

La disfunción del esfínter de Oddi es un síndrome clínico causado por una enfermedad funcional (discinesia) o estructural (estenosis) (4). Se caracteriza clínicamente por la presencia de dolor abdominal, similar al cólico biliar o dolor tipo pancreático en ausencia de patología biliopancreática orgánica. También se debe sospechar en pacientes con pancreatitis aguda recurrente idiopática; el dolor y la pancreatitis deben asociarse con elevación de enzimas pancreáticas o hepáticas, o dilatación del conducto biliar común o pancreático (5).

Este síndrome es más frecuente en mujeres colecistectomizadas entre los 20 y 50 años. La prevalencia estimada de disfunción del esfínter de Oddi de la población en general es del $1 \%$, que aumenta a $20 \%$ para pacientes con dolor persistente posterior a colecistectomía y a $70 \%$ en pacientes con pancreatitis aguda recurrente idiopática. Sin embargo, su verdadera prevalencia es difícil de determinar debido a la falta de biomarcadores definitivos o criterios diagnósticos (6-10).

Existen factores de riesgo implicados en la disfunción del esfínter de Oddi estructural que incluyen coledocolitiasis, colangiopancreatografía retrógrada endoscópica (CPRE) o manipulación quirúrgica de la vía biliar, colesterolosis, pancreatitis, ascaridiasis y enfermedad maligna (5).

Para el diagnóstico de disfunción del esfínter de Oddi existe la clasificación de Milwaukee que divide a los pacientes en 3 tipos según la presentación clínica, alteración en paraclínicos y en estudios de imagen (Tabla 1) (11-13). Sin embargo, en 2016 se publicó la clasificación de Roma IV (Tabla 2), que describe 2 tipos de disfunción del esfínter de Oddi biliar y el tercer tipo correspondiente a dolor abdominal funcional episódico (14).

En la literatura colombiana, hasta donde tenemos conocimiento, no existe ningún reporte de caso relacionado con la disfunción del esfínter de Oddi; además, existe controversia en el diagnóstico y tratamiento de esta patología.

Tabla 1. Clasificación de Milwaukee para disfunción del esfínter de Oddi (15)

Disfunción del esfínter de Oddi tipo biliar

\begin{tabular}{|c|c|}
\hline $\begin{array}{l}\text { Tipo I: } \\
\text { - Dolor tipo biliar } \\
\text { - Elevación de enzimas hepáticas (AST, ALT, FA) } 2 \text { veces por encima del } \\
\text { límite normal, documentado en al menos } 2 \text { oportunidades durante el } \\
\text { episodio de dolor } \\
\text { - Dilatación del colédoco (>12 mm) o retardo en el drenaje biliar (> } 45 \\
\text { min) }\end{array}$ & $\begin{array}{l}\text { Tipo I: } \\
\text { - Pancreatitis recurrente o dolor de posible origen pancreático } \\
\text { - Elevación de enzimas pancreáticas (amilasa, lipasa) } 2 \text { veces por } \\
\text { encima del límite normal, documentado en al menos } 2 \text { oportunidades } \\
\text { durante el episodio de dolor } \\
\text { - Dilatación del conducto pancreático (cabeza }>6 \mathrm{~mm} \text { y cuerpo }>5 \mathrm{~mm} \text { ) } \\
\text { o retardo en el drenaje pancreático (>9 min) }\end{array}$ \\
\hline $\begin{array}{l}\text { Tipo II: } \\
\text { - Dolor de tipo biliar y al menos } 1 \text { de los otros } 2 \text { criterios anotados }\end{array}$ & $\begin{array}{l}\text { Tipo II: } \\
\text { - Dolor de posible origen pancreático y al menos } 1 \text { de los otros } 2 \\
\text { criterios anotados }\end{array}$ \\
\hline $\begin{array}{l}\text { Tipo III: } \\
\text { - Dolor de tipo biliar sin ninguna otra alteración }\end{array}$ & $\begin{array}{l}\text { Tipo III: } \\
\text { - Dolor de posible origen pancreático sin ninguna otra alteración }\end{array}$ \\
\hline
\end{tabular}

ALT: alanina-aminotransferasa; AST: aspartato-aminotransferasa; FA: fosfatasa alcalina. Tomado de: Madura JA 2nd et al. Surg Clin North Am. 2007;87(6):1417-29, ix 
Tabla 2. Criterios de la disfunción del esfínter de Oddi de tipo biliar y pancreático (ROMA IV) (14)

Disfunción del esfinter de Oddi de tipo biliar

\section{Diagnóstico}

Debe incluir los siguientes criterios:

- Dolor de tipo biliar

- Elevación de enzimas hepáticas o dilatación del colédoco

- Ausencia de coledocolitiasis u otras anormalidades estructurales

Criterios de soporte:

- Amilasa y lipasa normales

- Manometría del esfínter de Oddi anormal

Gammagrafía hepatobiliar

Sospecha de disfunción del esfínter de Oddi de tipo biliar:

- Dolor de tipo biliar y al menos 1 hallazgo objetivo asociado

Dolor abdominal funcional episódico:

- Dolor de tipo biliar sin ninguna otra alteración
Disfunción del esfínter de Oddi de tipo pancreático

Diagnóstico

Debe incluir los siguientes criterios:

- Episodios recurrentes de pancreatitis documentados (dolor típico con amilasa o lipasa $>3$ veces el límite superior normal o estudio de imagen que evidencia pancreatitis aguda)

- Otras etiologías de pancreatitis excluidas

- USE negativa

- Manometría del esfínter de Oddi anormal

\section{CASO CLÍNICO}

Se trata de una mujer de 67 años con tratamiento extrainstitucional por colelitiasis a quien se le realizó una colecistectomía por laparotomía en 2013 y tenía antecedentes de dislipidemia en tratamiento con atorvastatina. Después del procedimiento quirúrgico, consultó reiteradamente al servicio de urgencias por dolor abdominal de predominio en el hipocondrio derecho tipo cólico asociado con emesis de características biliares y con hepatograma siempre dentro de límites normales. En diciembre de 2016 se realizó una colangiopancreatografía por resonancia nuclear magnética (CPRNM), que reportó la ausencia quirúrgica de la vesícula biliar y dilatación de la vía biliar intrahepática en el lóbulo izquierdo con colédoco de $8 \mathrm{~mm}$, sin coledocolitiasis (Figura 1). El hemograma y hepatograma estaban dentro de límites normales (Tabla 3).

En octubre de 2017 fue valorada en consulta de nuestro servicio y, por las características clínicas y el antecedente quirúrgico, se sospechó disfunción del esfínter de Oddi. Se ordenó una gammagrafía con ácido iminodiacético hepatobiliar (HIDA), que se realizó en noviembre de 2017 y reportó: "eliminación a la vía intrahepática antes de 15 minutos con abundante retención en los conductos hepáticos mayores y el colédoco. La cuantificación en la escala de Sostre para disfunción del esfínter de Oddi tuvo un puntaje de 9 puntos (son consistentes los puntajes $>5$ puntos)" (Tabla 4, Figura 2).

Con lo anterior se consideró una disfunción del esfínter de Oddi tipo II y se llevó a CPRE más esfinterotomía endoscópica en febrero de 2018, que documentó una papila mayor normal con vía biliar intra- y extrahepática dilatada

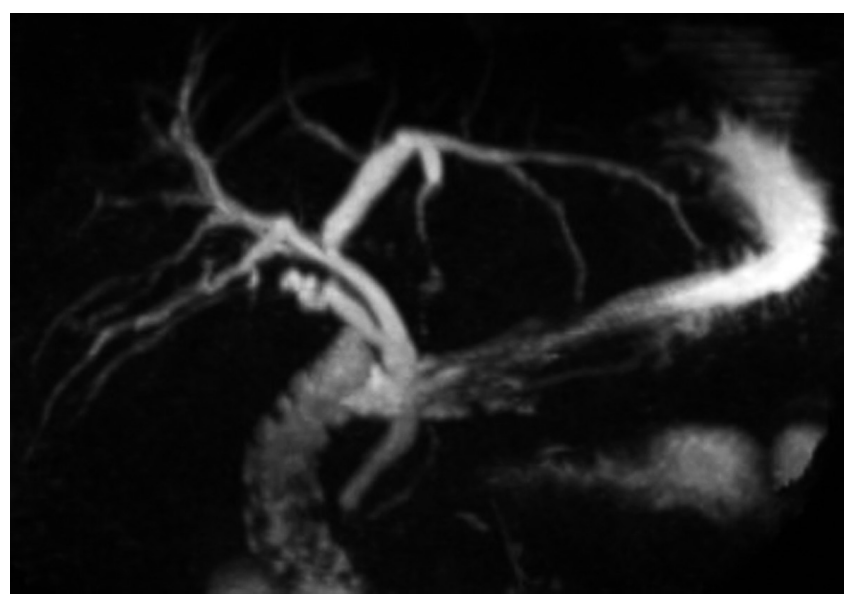

Figura 1. CPRNM. Estado poscolecistectomía, dilatación de la vía biliar intrahepática en el lóbulo izquierdo, colédoco de $8 \mathrm{~mm}$.

Tabla 3. Paraclínicos poscolecistectomía

\begin{tabular}{|ll}
\hline Paraclínicos & \multicolumn{1}{c}{ Resultados } \\
\hline Hemograma & - Leucocitos: $10100 \times 10^{3} / \mu \mathrm{L}$ \\
& - Neutrófilos: $7600 \times 10^{3} / \mu \mathrm{L}$ \\
& - Hemoglobina: $15,5 \mathrm{~g} / \mathrm{dL}$ \\
& - Hematocrito: $45,7 \%$ \\
& - Plaquetas: $307000 \times 10^{3} / \mu \mathrm{L}$ \\
Perfil hepático & - AST: $19 \mathrm{U} / \mathrm{L}$ \\
& - ALT: $36 \mathrm{U} / \mathrm{L}$ \\
& - Fosfatasa alcalina: $84 \mathrm{U} / \mathrm{L}$ \\
& - Bilirrubina total: $0,7 \mathrm{mg} / \mathrm{dL}$ \\
& - Bilirrubina directa: $0,3 \mathrm{mg} / \mathrm{dL}$ \\
& - Bilirrubina indirecta: $0,4 \mathrm{mg} / \mathrm{dL}$
\end{tabular}


Tabla 4. Puntaje en la escala de Sostre

\section{Disfunción del Oddi. Puntaje de Sostre}

\section{Puntos Paciente}

Tiempo de pico del colédoco

$-<10$ minutos

$>10$ minutos

0 1

Tiempo de visualización biliar

- $<15 \mathrm{~min}$

- > $15 \mathrm{~min}$

Prominencia del árbol biliar

- No prominente

Prominencia de conductos mayores

intrahepáticos

Prominencia de conductos intrahepáticos menores

Visualización intestinal

- < 15 minutos

- 15-30 minutos

$>30$ minutos

Evacuación del colédoco

- $>50 \%$

- $<50 \%$

Sin cambios

Aumenta

1

3

Relación colédoco/hígado

- Colédoco $60 \mathrm{~min} \leq$ hígado $60 \mathrm{~min}$

- Colédoco $60 \mathrm{~min}>$ hígado $60<$ hígado $15 \mathrm{~min}$

- Colédoco $60 \mathrm{~min}>$ hígado $60 \mathrm{e}=$ hígado $15 \mathrm{~min}$

- Colédoco $60 \mathrm{~min}>$ hígado 60 y > hígado $15 \mathrm{~min}$

Total

9

La cuantificación en la escala de Sostre para disfunción del esfínter de Oddi tuvo un puntaje de 9 puntos (son consistentes puntajes $>5$ puntos).

$(1,3 \mathrm{~cm})$. Se realizó esfinterotomía biliar y se exploró la vía biliar con canastilla hasta los hepáticos, obteniendo la salida de líquido biliar claro.

La evolución pos-CPRE fue satisfactoria y sin ninguna complicación. En febrero de 2020 (2 años después de la CPRE) se realizó el último control ambulatorio en el que la paciente se encontró asintomática y con un hepatograma normal. No volvió a requerir atención por el servicio de urgencias después de la CPRE.

\section{DISCUSIÓN}

Se presenta una paciente con sospecha de disfunción del esfínter de Oddi después de colecistectomía. Se soportó el diagnóstico con gammagrafía con HIDA, por lo cual se

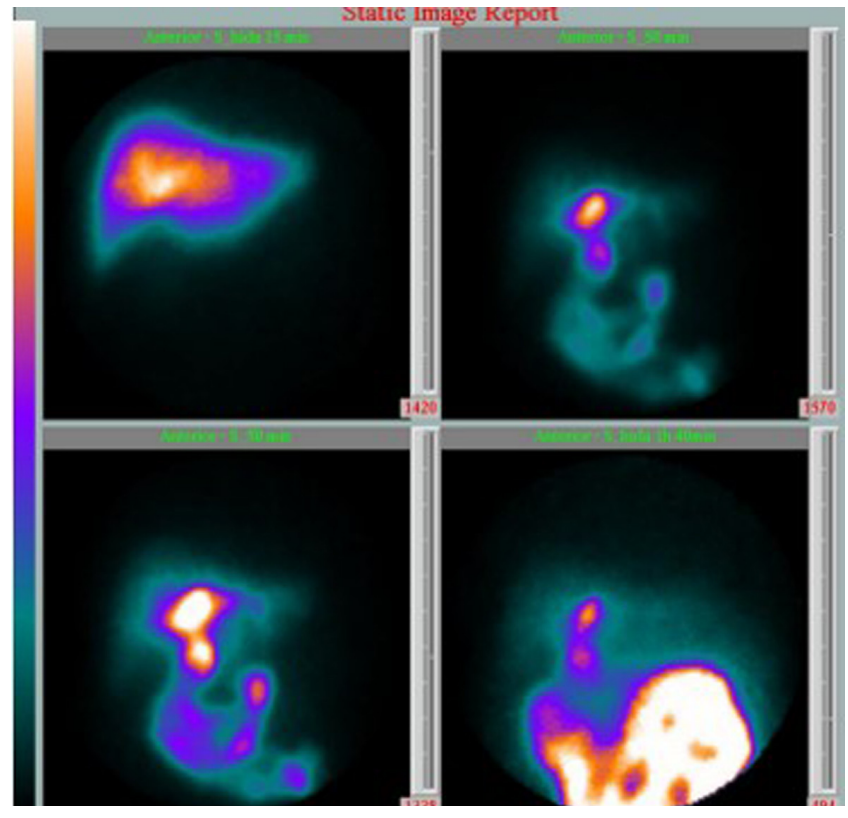

Figura 2. Gammagrafía con HIDA, eliminación a la vía intrahepática antes de 15 minutos con abundante retención en los conductos hepáticos mayores y el colédoco.

programó para CPRE con resolución del cuadro clínico posterior a la CPRE.

Clínicamente, es difícil diferenciar la disfunción del esfínter de Oddi de otras enfermedades biliopancreáticas, así como de otros trastornos digestivos funcionales; pero la historia clínica, el examen físico, los paraclínicos y los estudios de imagen deben evaluarse críticamente para sospechar el diagnóstico.

La remisión a centros expertos en patología biliopancreática es fundamental en el desenlace de esta entidad, donde previo análisis de la clínica del paciente, se deben ordenar los paraclínicos (enzimas hepáticas y pancreáticas) y estudios de imagen que incluyen ecografía abdominal, tomografía de abdomen, CPRNM, endosonografía biliopancreática (EUS) o técnicas isotópicas como la gammagrafía hepatobiliar (10).

Si se analiza el diagnóstico de disfunción del esfínter de Oddi, se puede anotar que, en pacientes con dilatación de la vía biliar, la sospecha diagnóstica se basa en la sintomatología y el hepatograma; cuando la probabilidad de patología biliar es alta y se han descartado otras causas por las imágenes anotadas previamente, se sugiere CPRE (16). Por el contrario, en presencia de dilatación de la vía biliar, pero con probabilidad baja de patología biliar (por sintomatología y hepatograma) se sugiere practicar USE o CPRNM, que son comparables en términos diagnósticos con la CPRE, pero sin la morbimortalidad asociada con 
la misma (16). Específicamente, si se analiza la USE en el contexto biliar, se encuentra que su rendimiento diagnóstico en pacientes con dilatación de la vía biliar y hepatograma anormal es solo del $53 \%$ (16) y disminuye al $6 \%$ en pacientes con dilatación de la vía biliar y hepatograma normal (16). Este $6 \%$ se asocia con condiciones como edad mayor a 65 años y estado poscolecistectomía (16).

La disfunción del esfínter de Oddi, en particular la estenosis papilar, puede causar dilatación del conducto biliar común sin ser diagnosticada por la USE. Inclusive en pacientes con dilatación de la vía biliar y una USE no diagnóstica, esta etiología debe considerarse (16).

La CPRE con la manometría del esfínter de Oddi se ha recomendado para el estudio de la disfunción del esfínter de Oddi. En pacientes con sospecha clínica y alguna alteración objetiva de patología biliar (disfunción del esfínter de Oddi tipo II), la elevación de la presión en reposo del esfínter de Oddi $>40 \mathrm{~mm}$ Hg es diagnóstica para la disfunción del esfínter de Oddi y es un buen predictor de la resolución de síntomas después de esfinterotomía endoscópica. Sin embargo, la posibilidad de pancreatitis pos-CPRE y manometría del esfínter de Oddi es hasta del $30 \%(11,17-19)$. En nuestra opinión, el nivel de riesgo anotado asociado con un estudio diagnóstico es inaceptable, sin contar con que existe una posibilidad de falsos negativos hasta del $65 \%(20,21)$.

Se han realizado investigaciones en métodos diagnósticos y terapéuticos que incluyen prueba de Nardi, CPRNM con secretina, gammagrafía con colecistoquinina, prueba de imagen luminal funcional, bloqueantes de los canales de calcio, antidepresivos tricíclicos, nitroglicerina, somatostatina y toxina botulínica. Ninguna de las anteriores alternativas ha logrado demostrar utilidad clínica relevante hasta la fecha (22-29).

Los pacientes con disfunción del esfínter de Oddi tipo I tienen indicación de esfinterotomía endoscópica sin necesidad de la realización de estudios adicionales $(20,30)$. Los pacientes con disfunción del esfínter de Oddi tipo II y III son de diagnóstico y manejo controvertidos. En nuestra opinión, todo paciente con sospecha de disfunción del esfínter de Oddi tipo II y III debe ser llevado a gammagrafía hepatobiliar pre-CPRE debido a que la especificidad del estudio de medicina nuclear es al menos del $90 \%$ (14, 31-34). Además, en la evaluación del dolor tipo biliar pos- colecistectomía, la gammagrafía está incluida en el algoritmo diagnóstico (14).

En los pacientes con disfunción del esfínter de Oddi tipo II, el tratamiento es la esfinterotomía endoscópica porque la respuesta clínica, a pesar de ser variable, en manos expertas es en general favorable, y en algunos reportes tiene éxito hasta del $90 \%$. No sugerimos realizar manometría del esfínter de Oddi previa por los ya mencionados riesgos de complicación y la discutible relación entre los hallazgos de la manometría del esfínter de Oddi y la disfunción del esfínter de Oddi (12, 35-37).

Los pacientes con disfunción del esfínter de Oddi tipo III son los más difíciles de diagnosticar debido a la ausencia de anormalidades de laboratorio e imagen, además de que su manejo es controvertido. Este tipo de pacientes no se beneficia de la realización de CPRE y esfinterotomía endoscópica (disminución de síntomas en aproximadamente el $20 \%$ ) (38-41); por el contrario, se someten a los riesgos inherentes a dicho procedimiento (42). Por lo anterior, el tratamiento para los pacientes con disfunción del esfínter de Oddi tipo III debe ser sintomático, basado en la farmacología utilizada para trastornos funcionales gastrointestinales (43).

En conclusión, sugerimos sospechar de disfunción del esfínter de Oddi en pacientes que después de la realización de colecistectomía presenten sintomatología compatible con dolor de tipo biliar o pancreático; así mismo, plantear la sospecha diagnóstica con herramientas diferentes a la manometría del esfínter de Oddi (gammagrafía) que permitan tomar, basados en su especificidad, la decisión de una CPRE más esfinterotomía endoscópica que resuelva la sintomatología del paciente.

Finalmente, este caso sirve como punto de partida para la realización de estudios prospectivos en otros grupos poblaciones.

\section{Conflictos de interés}

Los autores declaran no tener ningún conflicto de intereses.

\section{Fuente de financiación}

No hubo ninguna fuente de apoyo financiero.

\section{REFERENCIAS}

1. Avisse C, Flament JB, Delattre JF. Ampulla of Vater. Anatomic, embryologic, and surgical aspects. Surg Clin North Am. 2000;80(1):201-12. https://doi.org/10.1016/s0039-6109(05)70402-3
2. HAND BH. An anatomical study of the choledochoduodenal area. Br J Surg. 1963;50:486-94. https://doi.org/10.1002/bjs.18005022303

3. Guelrud M, Mendoza S, Rossiter G, Villegas MI. Sphincter of Oddi manometry in healthy volunteers. Dig Dis Sci. 
1990;35(1):38-46.

https://doi.org/10.1007/BF01537220

4. Behar J, Corazziari E, Guelrud M, Hogan W, Sherman

$S$, Toouli J. Functional gallbladder and sphincter of oddi disorders. Gastroenterology. 2006;130(5):1498-509. https://doi.org/10.1053/j.gastro.2005.11.063

5. Moody F, Poots J. Dysfunction of the ampulla of Vater. En: Braasch J, Tompkins R (editores). Surgical Diseases of the Biliary Tract and Pancreas, multidisciplinary management. St. Louis: Mosby; 1994. p. 334-48.

6. George J, Baillie J. Biliary and gallbladder dyskinesia. Curr Treat Options Gastroenterol. 2007;10(4):322-7. https://doi.org/10.1007/s11938-007-0075-2

7. Toouli J. What is sphincter of Oddi dysfunction? Gut. 1989;30(6):753-61. https://doi.org/10.1136/gut.30.6.753

8. Drossman DA, Li Z, Andruzzi E, Temple RD, Talley NJ, Thompson WG, Whitehead WE, Janssens J, Funch-Jensen P, Corazziari E, et al. U.S. householder survey of functional gastrointestinal disorders. Prevalence, sociodemography, and health impact. Dig Dis Sci. 1993;38(9):1569-80. https://doi.org/10.1007/BF01303162

9. Eversman D, Fogel EL, Rusche M, Sherman S, Lehman GA. Frequency of abnormal pancreatic and biliary sphincter manometry compared with clinical suspicion of sphincter of Oddi dysfunction. Gastrointest Endosc. 1999;50(5):637-41. https://doi.org/10.1016/s0016-5107(99)80011-x

10. Kaw M, Brodmerkel GJ Jr. ERCP, biliary crystal analysis, and sphincter of Oddi manometry in idiopathic recurrent pancreatitis. Gastrointest Endosc. 2002;55(2):157-62. https://doi.org/10.1067/mge.2002.118944

11. Hogan WJ, Geenen JE. Biliary dyskinesia. Endoscopy. 1988;20 Suppl 1:179-83. https://doi.org/10.1055/s-2007-1018172

12. Sherman S, Troiano FP, Hawes RH, O'Connor KW, Lehman GA. Frequency of abnormal sphincter of Oddi manometry compared with the clinical suspicion of sphincter of Oddi dysfunction. Am J Gastroenterol. 1991;86(5):586-90.

13. Solano J. Disfunción del esfínter de Oddi. En: Aponte D, Cañadas R (editores). Técnicas de endoscopia digestiva. 3. ${ }^{a}$ edición. Bogotá: Asociación Colombiana de Endoscopia Digestiva; 2018. p. 515-9.

14. Elta G, Cotton P, Carter C, Corraziari E, Pasricha P. Gallbladder and sphincter of Oddi Disorders. En: Drossman D (ed). Rome IV Functional gastrointestinal disorders. 4. ${ }^{a}$ edición. Raleigh: The Rome Foundation; 2016. p. 1117-78.

15. Madura JA 2nd, Madura JA. Diagnosis and management of sphincter of Oddi dysfunction and pancreas divisum. Surg Clin North Am. 2007;87(6):1417-29, ix. https://doi.org/10.1016/j.suc.2007.08.011

16. Malik S, Kaushik N, Khalid A, Bauer K, Brody D, Slivka A, McGrath K. EUS yield in evaluating biliary dilatation in patients with normal serum liver enzymes. Dig Dis Sci.
2007;52(2):508-12.

https://doi.org/10.1007/s10620-006-9582-6

17. Fogel EL, Eversman D, Jamidar P, Sherman S, Lehman GA. Sphincter of Oddi dysfunction: pancreaticobiliary sphincterotomy with pancreatic stent placement has a lower rate of pancreatitis than biliary sphincterotomy alone. Endoscopy. 2002;34(4):280-5. https://doi.org/10.1055/s-2002-23629

18. Steinberg WM. Controversies in clinical pancreatology: should the sphincter of Oddi be measured in patients with idiopathic recurrent acute pancreatitis, and should sphincterotomy be performed if the pressure is high? Pancreas. 2003;27(2):118-21. https://doi.org/10.1097/00006676-200308000-00002

19. Calzadilla J, Sanhueza N, Farías S, González F. Pancreatitis recurrente secundaria a disfunción del esfínter de Oddi: presentación de un caso [Recurrent pancreatitis secondary to sphincter of Oddi dysfunction: case report]. Medwave. 2016;16(9):e6585. https://doi.org/10.5867/medwave.2016.09.6585

20. Madura JA, Madura JA 2nd, Sherman S, Lehman GA. Surgical sphincteroplasty in 446 patients. Arch Surg. 2005; 140(5):504-11; discussion 511-3. https://doi.org/10.1001/archsurg.140.5.504

21. Cicala M, Habib FI, Vavassori P, Pallotta N, Schillaci O, Costamagna G, Guarino MP, Scopinaro F, Fiocca F, Torsoli A, Corazziari E. Outcome of endoscopic sphincterotomy in post cholecystectomy patients with sphincter of Oddi dysfunction as predicted by manometry and quantitative choledochoscintigraphy. Gut. 2002;50(5):665-8. https://doi.org/10.1136/gut.50.5.665

22. Nardi GL, Acosta JM. Papillitis as a cause of pancreatitis and abdominal pain: role of evocative test, operative pancreatography and histologic evaluation. Ann Surg. 1966;164(4):611-21. https://doi.org/10.1097/00000658-196610000-00008

23. Piccinni G, Angrisano A, Testini M, Bonomo GM. Diagnosing and treating Sphincter of Oddi dysfunction: a critical literature review and reevaluation. J Clin Gastroenterol. 2004;38(4):350-9. https://doi.org/10.1097/00004836-200404000-00010

24. Pereira SP, Gillams A, Sgouros SN, Webster GJ, Hatfield AR. Prospective comparison of secretin-stimulated magnetic resonance cholangiopancreatography with manometry in the diagnosis of sphincter of Oddi dysfunction types II and III. Gut. 2007;56(6):809-13. https://doi.org/10.1136/gut.2006.099267

25. Sharma BC, Agarwal DK, Dhiman RK, Baijal SS, Choudhuri G, Saraswat VA. Bile lithogenicity and gallbladder emptying in patients with microlithiasis: effect of bile acid therapy. Gastroenterology. 1998;115(1):124-8. https://doi.org/10.1016/s0016-5085(98)70373-7

26. Kunwald P, Drewes AM, Kjaer D, Gravesen FH, McMahon BP, Madácsy L, Funch-Jensen P, Gregersen H. A new distensibility technique to measure sphincter of Oddi function. 
Neurogastroenterol Motil. 2010;22(9):978-83, e253. https://doi.org/10.1111/j.1365-2982.2010.01531.x

27. Khuroo MS, Zargar SA, Yattoo GN. Efficacy of nifedipine therapy in patients with sphincter of Oddi dysfunction: a prospective, double-blind, randomized, placebo-controlled, cross over trial. Br J Clin Pharmacol. 1992;33(5):477-85. https://doi.org/10.1111/j.1365-2125.1992.tb04074.x

28. Sand J, Nordback I, Koskinen M, Matikainen M, Lindholm TS. Nifedipine for suspected type II sphincter of Oddi dyskinesia. Am J Gastroenterol. 1993;88(4):530-5.

29. Nakeeb A. Sphincter of Oddi dysfunction: how is it diagnosed? How is it classified? How do we treat it medically, endoscopically, and surgically? J Gastrointest Surg. 2013;17(9):1557-8. https://doi.org/10.1007/s11605-013-2280-8

30. Small AJ, Kozarek RA. Sphincter of Oddi Dysfunction. Gastrointest Endosc Clin N Am. 2015;25(4):749-63. https://doi.org/10.1016/j.giec.2015.06.009

31. Corazziari E, Cicala M, Scopinaro F, Schillaci O, Habib IF, Pallotta N. Scintigraphic assessment of SO dysfunction. Gut. 2003;52(11):1655-6. https://doi.org/10.1136/gut.52.11.1655

32. Craig AG, Peter D, Saccone GT, Ziesing P, Wycherley A, Toouli J. Scintigraphy versus manometry in patients with suspected biliary sphincter of Oddi dysfunction. Gut. 2003;52(3):352-7. https://doi.org/10.1136/gut.52.3.352

33. Sostre S, Kalloo AN, Spiegler EJ, Camargo EE, Wagner HN Jr. A noninvasive test of sphincter of Oddi dysfunction in postcholecystectomy patients: the scintigraphic score. J Nucl Med. 1992;33(6):1216-22.

34. Corazziari E, Cicala M, Habib FI, Scopinaro F, Fiocca F, Pallotta N, Viscardi A, Vignoni A, Torsoli A. Hepatoduodenal bile transit in cholecystectomized subjects. Relationship with sphincter of Oddi function and diagnostic value. Dig Dis Sci. 1994;39(9):1985-93. https://doi.org/10.1007/BF02088136
35. Sherman S. What is the role of ERCP in the setting of abdominal pain of pancreatic or biliary origin (suspected sphincter of Oddi dysfunction)? Gastrointest Endosc. 2002;56(6 Suppl):S258-66. https://doi.org/10.1067/mge.2002.129016

36. Geenen JE, Nash JA. The role of sphincter of Oddi manometry and biliary microscopy in evaluating idiopathic recurrent pancreatitis. Endoscopy. 1998;30(9):A237-41. https://doi.org/10.1055/s-2007-1001447

37. Petersen BT. Sphincter of Oddi dysfunction, part 2: Evidence-based review of the presentations, with "objective" pancreatic findings (types I and II) and of presumptive type III. Gastrointest Endosc. 2004;59(6):670-87. https://doi.org/10.1016/s0016-5107(04)00297-4

38. Baillie J. Sphincter of Oddi dysfunction. Curr Gastroenterol Rep. 2010;12(2):130-4. https://doi.org/10.1007/s11894-010-0096-1

39. Hyun JJ, Kozarek RA. Sphincter of Oddi dysfunction: sphincter of Oddi dysfunction or discordance? What is the state of the art in 2018? Curr Opin Gastroenterol. 2018;34(5):282-287. https://doi.org/10.1097/MOG.0000000000000455

40. Shah T, Zfass A, Schubert ML. Management of Sphincter of Oddi Dysfunction: Teaching an Old SOD New Tricks? Dig Dis Sci. 2016;61(9):2459-61. https://doi.org/10.1007/s10620-016-4252-9

41. Wilcox CM. Sphincter of Oddi dysfunction Type III: New studies suggest new approaches are needed. World J Gastroenterol. 2015;21(19):5755-61. https://doi.org/10.3748/wjg.v21.i19.5755

42. Peñaloza-Ramírez A, Leal-Buitrago C, RodríguezHernández A. Adverse events of ERCP at San José Hospital of Bogotá (Colombia). Rev Esp Enferm Dig. 2009; 101(12):837-49. https://doi.org/10.4321/s1130-01082009001200003

43. Pérez LV. Disfunción del esfínter de Oddi y trastornos biliares funcionales. Revista andaluza de patología digestiva. 2017;40(5):233-42. 